\title{
What produces shrinkage of apparent size for a moving object?
}

\author{
ATSUKI HIGASHIYAMA and KAORU TANAKA' \\ Institute of Human Sciences, College of Integrated Arts and Sciences, University of Osaka \\ Prefecture, Sakai 593
}

\begin{abstract}
An object moving in the frontal plane is perceived to shrink to a fraction of its stationary size, and the greater the velocity of the object, the greater the shrinkage of apparent size. This fact has been explained either by the shrinkage of effective retinal size of light emitted by the moving object or by the subjective Lorentz contraction in which apparent size of an object is assumed to diminish as the apparent velocity increases. To examine the validity of these theories, the 13 subjects made size- and velocity-matches for a moving target under each of 16 combinations of angular velocity, gaze state, and visual field. The greatest shrinkage of size was obtained for the object moving swiftly across a narrow visual field while the eyes gazed a stationary point, and the velocity matches were related to both angular velocity and gaze state but were not related to apparent size. All aspects of the results did not agree with the previous theories. Instead, effective duration for which the retinal image is formed at the fovea is shown to be critical for apparent size of the moving object.
\end{abstract}

Key words: shrinkage of apparent size, apparent velocity, Lorentz contraction, the AubertFleischl effect, eye movements, effective durations.

Although geometric optical illusions such as the Müller-Lyer illusion and the Delboeuf illusion are good examples to demonstrate that size of a stationary object is not perceived veridically, apparent size is not accurate even under usual conditions in everyday life. Kanizsa (1979) showed that an object, on which another object is superimposed, appears to shrink to $2-4 \%$, as compared with its apparent size obtained without such an obstacle. Restle (1970) and Restle and Merryman (1968) discovered shrinkage of apparent size for an object being close to larger objects.

More drastic change of apparent size is observed for a moving object. A number of studies (Ansbacher, 1944; Kaneko \& Uchikawa, 1993; Seya, 1951; Stanley, 1970; Tanaka, 1942a, b) showed that an object moving in the frontal plane is perceived to

\footnotetext{
K. Tanaka is now at the Kawachinagano City
} Hall. shrink to a fraction of its actual size, and the greater the velocity of the object, the greater the shrinkage of apparent size. In this study, we examined what produces such shrinkage of apparent size, by reporting an experiment and comparing previous theories

To explain the shrinkage of apparent size, Ansbacher (1944) assumed that the exposure of light emitted by the moving object makes a trapezoidal distribution in the retina, and only the parts receiving maximum stimulation (i.e., effective retinal parts) are processed and other parts are inhibited probably through contrast effect. Since the size of the effective retinal parts diminishes as the velocity of the image moving across the retina increases, apparent size of the moving object is assumed to diminish with an increase in the velocity of the image moving across the retina.

About 30 years later, Caelli (1981) and Caelli, Hoffman, and Lindman (1978) attempted to interpret the shrinkage of appar- 
ent size on the basis of analogy with Lorentz contraction in physics. Assuming an isomorphism between physical space and visual space, they proposed the subjective Lorentz contraction, in which apparent size of a moving object shrinks as compared with the stationary object and the apparent size diminishes curvilinearly as the apparent velocity of the object increases. A notable feature of the subjective Lorentz contraction model that should be distinguished from Ansbacher's explanation is that apparent size of an object is a function of its apparent velocity. The velocity of an image on the retina does not determine apparent size directly, but it only influences apparent size through apparent velocity.

The main purpose of this study was to examine the validity of the two explanations of the shrinkage of apparent size. Consider a simple situation where there is no or little eye movement, for example, by having subjects fixate a stationary point and, therefore, an increase in angular velocity of a moving object increases both the velocity of the image on the retina (i.e., retinal velocity) and the apparent velocity of the object. Since, in this situation, retinal velocity and apparent velocity vary simultaneously as the object moves, it is difficult to identify the genuine variable that determines apparent size directly. One way of overcoming this difficulty is to infer the variable statistically by the method of multiple regression and partial correlation (Cohen \& Cohen, 1975; Oyama, 1974, 1977). In this study, we asked subjects to judge both apparent size and apparent velocity of a moving object and then attempted to reveal causal connection among angular velocity, judged size, and judged velocity. If fast retinal velocity gave rise to shrinkage of apparent size, there would be a high partial correlation between angular velocity and judged size. If fast apparent velocity resulted in shrinkage of apparent size, there would be a high partial correlation between judged size and judged velocity.

In addition to angular velocity, two variables were examined to assess the general properties of apparent size and apparent velocity. One variable is the gaze state of the eyes: A moving target was tracked with eyes (tracking condition) and a moving target was observed while fixating a stationary point (fixation condition). Note that the retinal velocity of the target is generally slower in the tracking condition than in the fixation condition, because the eye position in the tracking condition varies continuously or successively to maintain the retinal image stationary at or near the fovea. Therefore, if the effective retinal image size of the target, as was suggested by Ansbacher, is inversely related to retinal velocity of the target, apparent size for the fixation condition is smaller than that for the tracking condition.

The gaze state of the eyes is related to apparent velocity as well. According to the Aubert-Fleischl effect, the velocity of a tracked object is perceived to be slower than that of a non-tracked object (Brown, 1931; Aubert, 1886, 1887; Fleischl, 1882; Teuber, 1960). If the Aubert-Fleischl effect is combined with the subjective Lorentz contraction, then apparent size for the fixation condition would be smaller than that for the tracking condition. However, without the AubertFleischl effect, there would not be such a difference in apparent size between the two conditions.

The size of the frame across which an object moves may also influence apparent size and apparent velocity of the moving object. Brown (1931) observed that the smaller the length of the frame, the greater the apparent velocity of the object. From a combination of the subjective Lorentz contraction and Brown's observation, it is suggested that even if the angular velocity remains constant, apparent size of a moving 
object is smaller when viewed with smaller frame. A number of studies (Anstis \& Atkinson, 1967; Haber \& Nathanson, 1968; Parks, 1965; Rock, Halper, DiVita, \& Wheeler, 1987) have indeed demonstrated that a target moving behind a narrow aperture appears in an opaque surface to shrink as compared with the stationary target seen directly without such an aperture. Algom and Cohen-Raz (1984), however, observed that apparent size for the target moving through long distance is perceived to be greater than that for the target moving through short distance. From this observation and the subjective Lorentz contraction, we can predict that an object viewed with smaller frame appears larger, because the small frame limits the moving distance of the target.

Method

\section{Subjects}

The subjects were 13 undergraduates who were not informed of the purpose of the experiment.

Apparatus

Figure 1 shows the perspective view of apparatus. Two horizontally moving rectangles $(8 \mathrm{~cm}$ high $\times 32 \mathrm{~cm}$ wide) were projected on the half-circular screen $(41 \mathrm{~cm}$ high), with a curvature radius of $100 \mathrm{~cm}$. Each rectangle was produced by a mirror, which rotated at a distance of $100 \mathrm{~cm}$ from the screen and

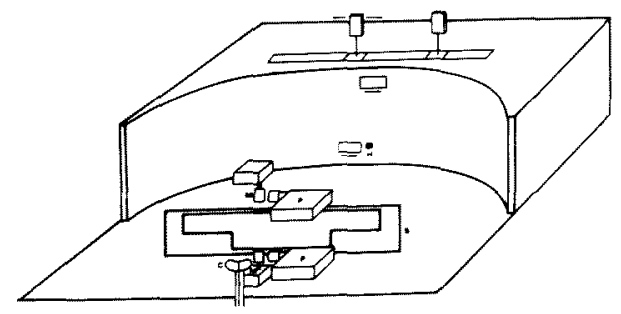

Figure 1. The perspective view of the apparatus. C: a chin rest; P: a projector; $M$ : a mirror; $\mathrm{H}$ : a fixation hole; $\mathrm{S}$ : screen limiting the visual field. reflected the light that was emitted by a Kodak Ektagraphic slide projector (Model AF-2). The rotating velocities of the lower mirror that projected the standard rectangle were $.34, .63, .77$, and $1.00 \mathrm{rps}$ and the rotating velocity of the upper mirror that projected the variable rectangle was controlled by the experimenter under the direction of the subject. Since the distance between the center of the screen and the eyes of the subject was $120 \mathrm{~cm}$, angular velocities of the standard seen by the subject were about 102, 189, 219 , and $301 \%$ at the center of the screen. The target always moved from the left to the right on the screen. The inner edges of the rectangles were separated by a distance of about $20 \mathrm{~cm}$. The horizontal eye level of the subject was positioned at the center of the two rectangles.

Above the screen, there was an optical bench, on which two boards $(10 \mathrm{~cm}$ high $\times$ $3.8 \mathrm{~cm}$ wide) were mounted. The right board was kept stationary and the left board was adjustable along the bench by the experimenter. The distance between inner edges of the boards was used to indicate apparent size of the standard rectangle.

The visual field of the subject was limited by an opaque screen at a distance of $26 \mathrm{~cm}$ from the subject. At the center of the opaque screen, there was an aperture through which the subject viewed the targets. The lateral size of the window was so changed that the standard could be viewed in a visual field of $20^{\circ}$ or $90^{\circ}$ and the variable could be viewed in a visual field of $90^{\circ}$. Since the center of the target screen was positioned $94 \mathrm{~cm}$ behind the aperture, the subjects recognized that the target screen and the aperture were not in the same plane.

There was a small window $(2 \mathrm{~cm}$ high $x$ $2 \mathrm{~cm}$ wide) straight ahead of the subject, on the movement path of the lower standard rectangle. A CRT was embedded in the wall behind the window to generate a series of 
two-digit random numbers by a computer at a speed of 1.52 numbers per second. These random numbers were used to have the subject fixate the center of the screen.

\section{Procedure}

The subject sat on a chair with his or her head on the chin rest, without usage of a biting board. The observation was binocular through the experiment. There were 16 conditions that resulted in by combining four velocities, two fields, and two gazes. For each trial, the standard rotated around the subject five times from the left to the right. When the standard rotated, the subject in the tracking condition was encouraged to follow up the standard with the eyes. On the other hand, the subject in the fixation condition was asked to read aloud the random numbers that emerged in succession on the CRT. While the subject failed to read the number, the trial was repeated immediately. In both conditions, the subject was instructed to memorize the apparent size and apparent velocity of the standard and to reproduce the size and velocity in the later phase.

After the standard was presented, the subject viewed the variable on the screen for velocity comparison and viewed the two boards on the bench for size comparison. For velocity comparison, the subject was asked to make the sign of stop when the velocity of the variable appeared to equal the remembered velocity of the standard. For each standard, there was one ascending trial, in which the velocity of the variable was gradually increased by the experimenter from the velocity that appeared to be definitely slower than the standard, and one descending trial, in which the velocity of the variable was decreased from the velocity that appeared to be definitely greater than the standard.

For size comparison, the subject made the sign of stop when the spatial separation between the inner edges of the two boards appeared to equal the remembered size of the standard. For each standard, there was one ascending trial, in which the size of the variable was increased gradually by the experimenter from the size that appeared to be definitely smaller than the standard, and one descending trial, in which the size of the variable was decreased from the size that appeared to be definitely greater than the standard.

For half the subjects, the tracking condition followed the fixation condition and for the other subjects, the tracking condition preceded the fixation condition. For each gaze condition, half the subjects made the size comparison first and then the velocity comparison, and the remaining subjects followed the reverse order.

\section{Results}

Figure 2 shows the results of size matches. The abscissa represents the angular velocity of the standard and the ordinate represents the matched size of the variable. The parameters are the gaze and field. Each data point is based on a mean of the 13 subjects.

The size matches were analyzed by a four-way (four velocities $x$ two gazes $x$ two

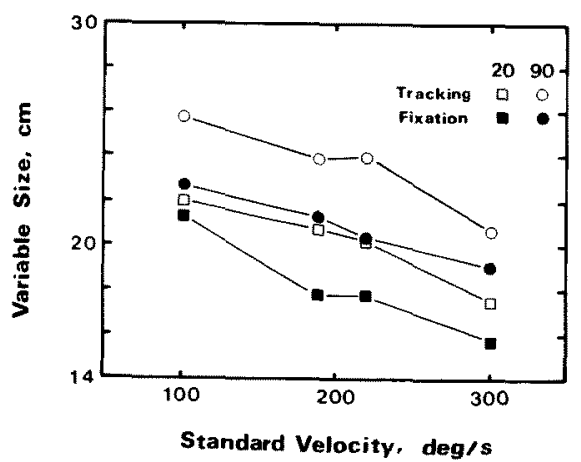

Figure 2. Size matches (in $\mathrm{cm}$ ) of the variable as a function of angular velocity of the standard (in $\%$ ). The circles represent the field size of $90^{\circ}$ and the squares represent the field size of $20^{\circ}$. The open marks represent the tracking condition and the filled marks represent the fixation condition. 
fields $\times$ two series) ANOVA for repeated measures. The main effect of velocity was significant, $F(3,36)=25.3, p<.001$, suggesting the size matches to decrease as the velocity increases. The size matches for the tracking condition were significantly larger than those for the fixation condition, $F(3$, $12)=18.2, p<.01$. The size matches for the large field were significantly larger than those for the small field, $F(3,12)=78.1$, $p<.001$. The main effect of series was not significant. No two-, three-. or four-way interaction was significant.

The velocity matches were analyzed by a four-way ANOVA for repeated measures. The main effect of series was significant, $F(1,12)=19.0 . p<.001$. The mean angular velocity was $195 \%$ for the ascending series and was $208 \% \mathrm{~s}$ for the descending series. The main effect of velocity was significant, $F(3,36)=533.4, p<.001$, indicating the velocity matches to increase as the angular velocity increases. The interaction of velocity with gaze was significant, $F(3,36)=6.3$, $p<.01$. Figure 3 shows the interaction graphically. The abscissa represents the angular velocity of the standard and the ordinate represents the mean angular velocity of the variable. The parameter is the gaze condition. Each data point is based on a mean taken

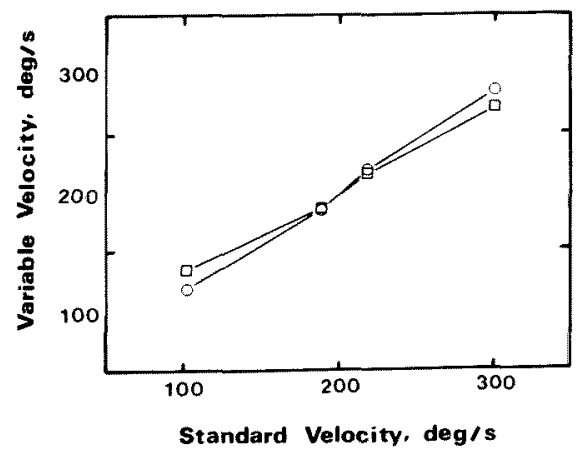

Figure 3. Velocity matches of the variable (in $\% / s$ ) as a function of angular velocity of the standard (in $\%$ ). The circles represent the tracking condition and the squares represent the fixation condition. across the subjects and the field conditions. It is suggested from Figure 3 that under the low velocity condition, the tracked target was perceived to be slower than the non-tracked target, but under the high velocity condition, the tracked target was perceived to be faster than the non-tracked target.

Figure 4 shows the size matches plotted against the velocity matches. The parameters are the standard velocity, field size, and gaze. The multiple regression equation for estimating size matches $(S)$ from velocity matches $(V)$, standard velocity $(V)$, field size $(F)$, and gaze $(G)$ was obtained by a least-squares criterion:

$$
\begin{aligned}
S^{\prime}= & 21.594+0.003 V^{\prime}-0.026 V+0.044 F \\
& +2.342 G,
\end{aligned}
$$

where gaze was coded 1 for the tracking condition and 0 for the fixation condition. The multiple regression coefficient of the four variables with size matches was .982 , accounting for $96.5 \%$ of the variance in size matches.

Table 1 shows the simple, semipartial, and partial correlations of size matches with the four variables. The partial correlations for field, $t(11)=9.31, p<.01$, and gaze, $t(11)$ $=7.97, p<.01$, were significantly different from zero, but the partial correlations for velocity matches and standard velocity were not. According to the results of semipartial

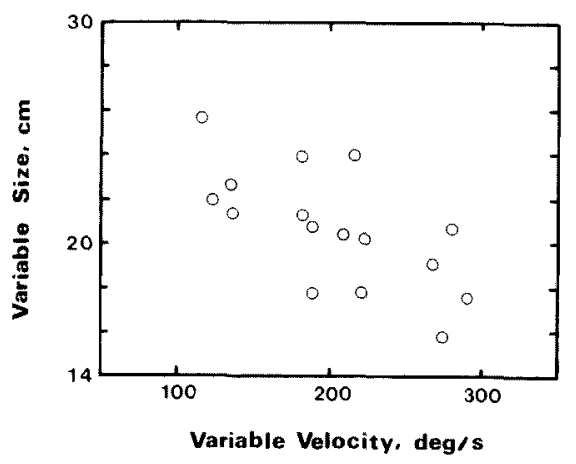

Figure 4. Size matches (in $\mathrm{cm}$ ) of the variable as a function of angular velocity of the variable (in $\%$ ). 
Table 1

Simple, semipartial, and partial correlations of size estimates with other four variables (i. e., velocity matches, standard velocity, field size, and gaze)

\begin{tabular}{lccc}
\hline & \multicolumn{3}{c}{ Correlation } \\
\cline { 2 - 4 } \multicolumn{1}{c}{ Variable } & Simple & Semipartial & Partial \\
\hline Velocity matches & $-.666^{* *}$ & .009 & .045 \\
Standard velocity & $-.637^{* *}$ & -.086 & -.408 \\
Field size & $-.592^{*}$ & $.525^{*}$ & $.939^{* *}$ \\
Gaze & .454 & .451 & $.920^{* *}$
\end{tabular}

$*: p<.05, * *: p<.01$.

correlation, field accounted uniquely for $27.6 \%$ (squared semipartial correlation) of the variance in size matches and gaze accounted uniquely for $20.4 \%$ of the variance. Thus, the field and gaze conditions are suggested to influence size matches directly but the apparent and angular velocities of the standard are not.

Similarly, the multiple regression equation for estimating velocity matches was also obtained from other four variables:

$$
\begin{aligned}
V^{\prime}= & 39.464+0.606 S^{\prime}+0.777 V-0.129 F \\
& -0.834 G
\end{aligned}
$$

The multiple regression coefficient was .993 , indicating that the explanatory variables together accounted for $98.5 \%$ of the variance in velocity matches. Table 2 shows the simple, semipartial, and partial correlations of velocity matches with the four variables. Standard velocity had a significantly high partial correlation with velocity matches, $t(11)=8.00, p<.01$, accounting uniquely for $8.7 \%$ of the variance in velocity matches. Thus, the standard velocity is suggested to affect the velocity matches directly, but other three variables are not.

\section{General Discussion}

Clearly, standard velocity, gaze state, and field size affected the size matches systemati-
Table 2

Simple, semipartial, and partial correlations of velocity estimates with other four variables (i. e., size matches, standard velocity, field size, and gaze)

\begin{tabular}{lccc}
\hline & \multicolumn{3}{c}{ Correlation } \\
\cline { 2 - 4 } \multicolumn{1}{c}{ Variable } & Simple & Semipartial & Partial \\
\hline Size matches & $-.666^{* *}$ & .006 & .045 \\
Standard velocity & $.990^{* *}$ & .296 & $.924^{* *}$ \\
Field size & -.065 & -.026 & -.206 \\
Gaze & .005 & -.003 &. .025
\end{tabular}

**: $p<, 01$

cally. That is, (1) as the velocity of the target increased, the size matches decreased, (2) the size matches for the tracked targets were larger than those for the non-tracked targets, and (3) a small visual field reduced size matches much more than did a large visual field.

As the angular velocity of the target increased, the velocity matches generally increased. This result is in agreement with a number of studies (Algom \& Cohen-Raz, 1984; Eisler \& Ottander, 1963; Semb, 1969; Kennedy, Yessenow, \& Wendt, 1972). Note, also, that the rate of increase in velocity matches was steeper for the tracked target than for the non-tracked target. In other words, the Aubert-Fleischl effect held true only under the low velocity condition, but not under the high velocity condition.

Field size did not influence velocity matches. This result may challenge the Brown study (1931) which suggested greater apparent velocity for an object viewed through smaller visual field. Since, in this experiment, the aperture that delimited field size and the screen on which the target moved were not perceived in the same plane, it seems that the effect of field size on apparent velocity was not at work or weakened. It should also be noted that the subjects in this experiment could make use of oculomotor 
cues and binocular disparity to perceive distances to the target and frame. Brown indeed showed that even when the target and frame are coplanar, the effects of frame are much weakened under binocular vision in a well-lit room.

\section{Failure of Previous Theories}

Subjective velocity theory. The results of the partial correlation analysis are useful to examine validity of the subjective Lorentz contraction. It was obtained that (1) the size matches were determined directly by both gaze state and field size, but were not determined directly by either apparent and angular velocity of the target, (2) the velocity matches were determined mainly by standard velocity, but not determined directly by gaze state and field size, and (3) there was no high partial correlation between the size matches and the velocity matches, although there was an intermediate simple correlation between them. Thus, apparent size and apparent velocity were determined separately by stimulus variables (i.e.. angular velocity and field size) and observation mode (i.e., gaze condition), and apparent size was not determined directly by apparent velocity. Clearly these results do not support the subjective Lorentz contraction.

The evidence against the subjective Lorentz contraction was also obtained from the serial effect that was found for velocity matches, but not for size matches. If, as is suggested by the subjective Lorentz contraction, the change of apparent size was contingent to the change of apparent velocity, then the serial effect would be found for both size and velocity matches or would not be found for either. The appearance of the serial effect only for velocity matches, therefore, may disprove the subjective Lorentz contraction hypothesis.

Retinal velocity theory. The shrinkage of size matches may be due partly to the effective retinal size of the target, which is assumed to be smallest for the rapidly moving target under the fixation condition and to be greatest for the slowly moving object under the tracking condition. However, since the effective retinal size depends only on the retinal velocity of the target that is determined by both angular velocity and gaze state, it is difficult to explain why the size matches were greatly reduced for small field size. rather than for large field size.

Eye movement theory. Mack and Herman (1972) compared the apparent distance through which a small luminous target appears to move with the apparent distance between two successively lit targets. In the former condition, the target was seen continuously with pursuit eye movement, whereas in the latter, the targets were seen with saccadic eye movement. From this finding, the shrinkage of apparent distance was considered to be due to saccadic eye movement. Coren, Bradley, Hoenig, and Girgus (1975) interpreted this shrinkage of apparent distance on the basis of the finding that the eye, which pursuits a moving target, lags a degree or more behind the target.

It is possible that the shrinkage of apparent size is generated by eye movement. To illustrate how saccadic eye movement exerts in perceiving size of a moving target, consider the condition where, while the eyes are directed to an arbitrary point $O$, the leading and trailing edges of the moving target are at the points $\mathrm{A}$ and $\mathrm{B}$, respectively, at a given moment. In this condition, if the angular distance between $\mathrm{O}$ and $\mathrm{A}$ is defined as $a$ and the angular distance between $\mathrm{O}$ and $\mathrm{B}$ as $b$, then the angular size of this target is $a-b$. If the eyes are allowed to pursuit the target but lag behind the target at the rate of $n(0<n<1)$, then angular magnitudes of saccadic eye movement for the angular distance $a$ and $b$ shrinks to $n a$ and $n b$, respectively, and consequently the angular size $a-b$ appears to shrink to $n(a-b)$. Despite this successful pre- 
diction, the weakest point of the eye movement theory is that apparent size shrank as well for the fixation condition where saccadic eye movement was suppressed.

\section{A Tentative Theory: Effective Duration}

The final theory to be able to explain the shrinkage of apparent size is that the shorter the duration to process the retinal image size of the target effectively, the smaller the apparent size of the target. The effective duration $t$ is assumed to be

$$
t=\frac{m F}{V} \text {, }
$$

where $V$ is the angular velocity, $F$ is the field size, and $m$ is a constant $(0 \leq m \leq 1)$. The $m$ value represents the degree to which the retinal image is formed at the fovea. If the retinal image is formed neatly at the fovea, the $m$ value is then unity, whereas if the retinal image is formed off the fovea, this value is less than unity. For the tracking condition where the retinal image of the target was maintained stationary at or near the fovea, the $m$ value is assumed to be unity. For the fixation condition where the retinal image moved over the retina, the retinal image was first at the peripheral, then at the fovea, and again at the peripheral. This leads us to assume that the $m$ value is somewhere between zero and unity.

Figure 5 shows the size estimates as a

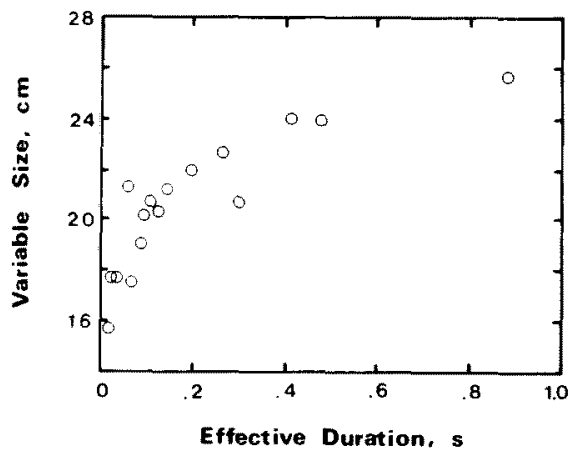

Figure 5. Size estimates (in $\mathrm{cm}$ ) as a function of effective duration (in s). function of effective duration of a moving target. In computing the effective duration, $m$ is assumed to be 1.0 for the tracking condition and 0.3 for the fixation condition. The $m$ value for the fixation condition was determined graphically by trial and error, so that a monotonically increasing function holds as much as possible between size estimate and effective duration.

Figure 5 indicates that the size matches increase with negative acceleration as the effective duration increases. If the retinal image of a moving target stimulates the fovea for more than $1 \mathrm{~s}$, the size matches approach the objective size, while it stimulates the fovea for less than $0.1 \mathrm{~s}$, the size matches is about half as large as the objective size. This dependence of apparent size on effective duration may remind us of the Gelb or $\tau$ effect (Helson, 1930; Helson \& King, 1931), which means that apparent distance between two successively lit targets diminishes greatly for the interstimulus interval that may produce optimal apparent movement. According to some reviews (Anstis, 1978, 1986; Kolers, 1972), an interstimulus interval for optimal apparent movement ranges from 40 to 200 $\mathrm{ms}$, depending on target duration, spatial separation between targets, and target intensity. Although apparent movement may not be equivalent to real movement, it should be noted that similar shrinkage of apparent size is obtained for both real movement and apparent movement.

\section{References}

Algom, A., \& Cohen-Raz, L. 1984 Visual velocity input-output functions: The integration of distance and duration onto subjective velocity. Journal of Experimental Psychology: Human Perception and Performance, 10, 486-501.

Ansbacher, H. L. 1944 Distortion in the perception of real movement. Journal of Experimental Psychology, 44, 1-23.

Anstis, S. M. 1978 Apparent movement. In R. H. Held \& H. W. Leibowitz (Eds.), Handbook of 
sensory physiology. Vol. 8. New York: SpringerVerlag. Pp. 655-673.

Anstis, S. M. 1986 Motion perception in the frontal plane: Sensory aspects. In K. R. Boff, L. Kaufman \& J. P. Thomas (Eds.), Handbook of perception and human performance. Vol. 1. Sensory processes and perception. New York: John Wiley and Sons. Chapter 16.

Anstis, S. M.. \& Atkinson, J. 1967 Distortions in moving figures viewed through a stationary slit. American Journal of Psychology. 80, 572-583.

Aubert. H. 1886 Die Bewegungsemptindung. Pfligers Archir fiir die gesamte Physiologie des Menschen und der Tiere, 39, 347-370.

Aubert, H. 1887 Die Bewegungsempfindung. Pfliggers Archiv fuir die gesamte Physiologie des Menschen und der Tiere, 40, 459-480.

Brown. J.F. 1931 The visual perception of velocity. Psychologische Forschung, 14, 199-232

Caelli. T. 1981 On the spatio-temporal determinants of some motion effects. Acta Psychologica, 48, 175-185.

Caelli, T., Hoffman, W., \& Lindman, H. 1978 Subjective Lorentz transformations and the perception of motion. Journal of the Optical Society of America, 68, 402-411.

Cohen, J., \& Cohen, P. 1975 Applied multiple regression/correlation analysis for the behavioral sciences. New York: John Wiley \& Sons.

Coren, S. Bradley, D. R., Hoenig, P., \& Girgus, J. S. 1975 The effect of smooth tracking and saccadic eye movements on the perception of size: The shrinking circle illusion. Vision Research, 15. 49-55.

Eisler, H., \& Ottander, C. 1963 On the problem of hysteresis in psychophysics. Journal of Experimental Psychology, 65, 530-536.

Fleischl, E. von. 1882 Physiologisch-optische Notizen. Sitzungsberichte der Akademie der Wissenschaften in Wien. MathematischNaturwissenschaftliche Klasse. Abteilung 3, Anatomie und Physiologie des Menschen und der Tiere, 86, 17-25.

Haber, R. N., \& Nathanson, L. S. 1968 Post-retinal storage? Some further observations on Parks' camel as seen through the eye of a needle. Perception \& Psychophysics, 3, 349-355.

Helson, H. 1930 The tau effect-an example of psychological relativety. Science, 71, 536-557.

Helson, H., \& King, S. M. 1931 The tau effect: An example of psychological relativity. Journal of Experimental Psychology, 14, 202-217.

Kaneko, H., \& Uchikawa, K. 1993 Apparent rela- tive size and depth of moving objects Perception. 22, 537-547.

Kanizsa, G. 1979 Organization in vision: Essays on Gestalt perception. New York: Praeger. Chapter 10.

Kennedy, R. S.. Yessenow, M. D., \& Wendt, G. R. 1972 Magnitude estimation of visual velocity. Journal of Psychology, 82, 133-144.

Kolers, P. A. 1972 Aspects of motion perception. Oxford: Pergamon Press.

Mack, A., \& Herman, E. 1972 A new illusion: The underestimation of distance during pursuit eye movements. Perception \& Psychophysics, 12, 471-473.

Oyama, T. 1974 Inferences in perception of space and motion. Psychologia, 17, 166-178.

Oyama, T. 1977 Analysis of causal relations in the perceptual constancies. In W. Epstein (Ed.), Stability and constancy in visual perception. New York: Wiley. Pp. 183-216.

Parks, T. 1965 Post-retinal visual storage. American Journal of Psychology, 78, 145-147.

Restle, F. 1970 Moon illusion explained on the basis of relative size. Science, 167, 1092-1096.

Restle, F., \& Merryman, C. T. 1968 An adaptationlevel theory account of a relative-size illusion. Psychonomic Science, 12, 229-230

Rock, I., Halper, F., DiVita, J., \& Wheeler, D. 1987 Eye movement as a cue to figure motion in anorthoscopis perception. Journal of Experimental Psychology: Human Perception and Performance, 13, 344-352.

Semb, G. 1969 Scaling automobile speed. Perception \& Psychophysics, 5, 97-101.

Seya, M. 1951 Undo-kido no genshoteki nagasa (On the apparent length of the path of real movememt). Japanese Journal of Psychology, 21, 113.

Stanley, G. 1970 Static visual noise and the Ansbacher effect. Quarterly Joumal of Experimental Psychology, 22, 43-48.

Tanaka, Y. 1942a Undo suru zukei no chikaku. Sono 1 (Perception of moving figures: 1). Japanese Journal of Psychology, 17, 333-352.

Tanaka, Y. 1942b Undo suru zukei no chikaku. Sono 2 (Perception of moving figures: 2 ). Japanese Journal of Psychology, 18, 443-458.

Teuber, H. -L. 1960 Perception. In J. Field, H. W. Magoun \& V. E. Hall (Eds.), Handbook of physiology, Section 1: Neurophysiology. Vol. 3. Washington, D. C.: American Physiological Society. Pp. 1595-1668.

(Received Oct. 20, 1993; accepted May 7, 1994) 